\title{
Gamma Knife Radiosurgery for Acoustic Schwannoma: Early Effects and Preservation of Hearing
}

\author{
Masafumi Hirato, Hiroshi InOUe, Masaru NaKamura, Chihiro OHYE, \\ Junko HIRATO*, Tohru SHIBAZAKI**, and Yoshitaka ANDOU** \\ Departments of Neurosurgery and *Pathology, Gunma University School of Medicine, \\ Maebashi; ${ }^{* *}$ Department of Neurosurgery, Hidaka Hospital, Takasaki, Gunma
}

\begin{abstract}
The effects of relatively low dose gamma knife irradiation on acoustic schwannoma were evaluated. The signal intensity change and tumor shrinkage on magnetic resonance (MR) images, change in hearing, and complications in 28 patients (mean age $47.0 \pm 13.6 \mathrm{yrs}$ ) were studied. Three patients had bilateral tumors. Six were already deaf when treated. The maximum tumor diameter was $35 \mathrm{~mm}$. The mean dose delivered to the tumor was $12.1 \pm 1.6 \mathrm{~Gy}$ at the periphery, and $25.2 \pm 4.3 \mathrm{~Gy}$ at the center. The mean follow-up time was 16 months and the longest 24 months. Lowering of the MR signal intensity in the tumor center appeared after 3 months at earliest but generally after 6 months. Signs of tumor shrinkage appeared within 12 months on average. Cyst in the tumor enlarged rapidly after treatment in two patients. The percentage of hearing preservation was $85 \%(17 / 20)$ at 3 months, $80 \%(16 / 20)$ at 6 months, $72 \%(13 / 18)$ at 9 months, $75 \%(12 / 16)$ at 12 months, $67 \%(8 / 12)$ at 15 months, $60 \%(6 / 10)$ at 18 months, and $50 \%(2 / 4)$ at 24 months. Subtle changes in hearing were detected by speech tone audiometry. Temporary facial numbness and weakness was seen in one patient each. No patient had lower cranial nerve paresis. Relatively low dose gamma knife radiosurgery is effective in suppressing growth of acoustic schwannoma with preservation of hearing.
\end{abstract}

Key words: acoustic schwannoma, gamma knife, radiosurgery, hearing preservation

\section{Introduction}

Gamma knife is now widely used for the treatment of acoustic tumors, ${ }^{6,7,9,10,12,13,15)}$ not only in elderly patients, but also patients with medical problems, bilateral tumors, a tumor in the only functioning ear, or a recurrent tumor and those who refuse surgical removal. Comparison of the results of radiosurgery with the results of microsurgery suggests that radiosurgery is the treatment of choice. ${ }^{13,15)}$ Acoustic schwannoma has been treated by gamma knife radiosurgery with a relatively high radiation dose,,$^{6,7,9,10)}$ but lower doses have been recommended. ${ }^{11,15)}$

We report our experience in treating acoustic schwannoma with a relatively low radiation dose based on changes in the tumor appearance on magnetic resonance (MR) images and the clinical course, especially changes in hearing acuity.

Received April 11, 1994; Accepted February 27, 1995

\section{Subjects and Methods}

Twenty-eight patients, 10 males and 18 females aged between 24 and 73 years old (mean \pm SD $47.0 \pm$ 13.6), were treated from May 1991 to December 1992. Three patients had bilateral tumors. The maximum lesion diameter was $35 \mathrm{~mm}$. Six patients had lost hearing acuity on the side of the tumor. Dosimetry was carefully planned on both axial and coronal MR images, especially at the porus of the internal auditory meatus, using computed tomographic (CT) scans to correct the distortion of the MR images. The mean isodose at the periphery was $48.9 \pm 6.3 \%$. The dose delivered to the tumor periphery was mean $12.1 \pm 1.6 \mathrm{~Gy}$, range $10-17 \mathrm{~Gy}$, and to the center mean $25.2 \pm 4.3 \mathrm{~Gy}$, range $17-34$ Gy. The time necessary for one fraction was usually between 4 and 7 minutes. The number of fractions was $5.0 \pm 2.1$. The mean follow-up time was 16 months and the longest was 24 months.

Three correlations were studied: when the signal change or shrinkage of the tumor was first observed 
on MR images, original tumor size (approximate volume), and the peripheral or center dose; the time course of hearing change (or cranial nerve paresis), by correlating the change in auditory acuity, original tumor size, and the dose delivered to the periphery or center, or the dose rate in the periphery; and the change in auditory acuity, the length of acoustic nerve irradiated which is related to the pons-petrus distance, and the dose delivered to the periphery.

The tumor was surgically removed in two patients at 3 and 4 months after gamma knife radiosurgery, because the cyst in the tumor had enlarged, although the solid part had shrunk. Histological examination of surgical specimens was possible in one of these two cases.

\section{Results}

\section{MR imaging study}

The MR signal intensity of the central area of the tumor had lowered in $17(65 \%)$ of the 26 patients excluding the two receiving surgical removal, starting at 3 months at earliest but generally at around 6 months (mean 5.7 mos). Shrinkage of the tumor appeared in six (23\%) of 26 patients, beginning at 12 months on average. Four patients showed both tumor shrinkage and lowering of the MR signal intensity in the central area. Two patients showed shrinkage of the tumor without change in the MR signal intensity. In seven $(27 \%)$ of 26 patients, the MR signal intensity and the tumor size remained unchanged (Fig. 1).

Larger tumors were associated with tumor shrinkage and more rapid appearance of signal changes. There was no definite correlation between

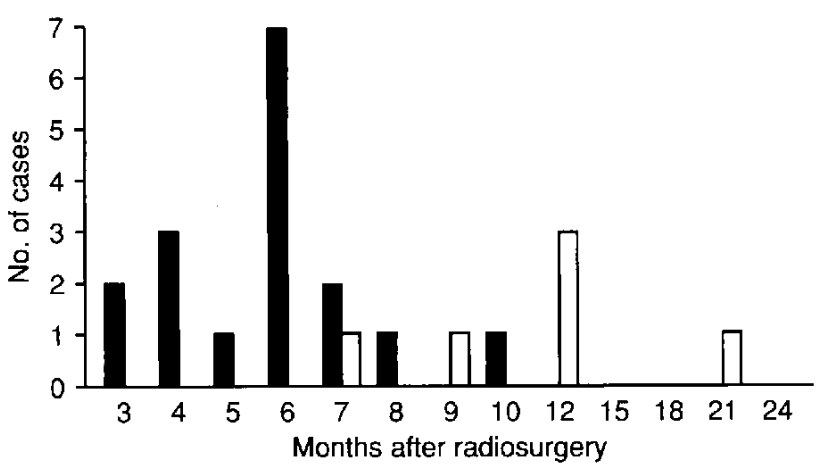

Fig. 1 Onset of MR signal intensity changes in the central area and tumor shrinkage. Closed bars show patients with lowering of the MR signal intensity. Open bars represent patients with tumor shrinkage.

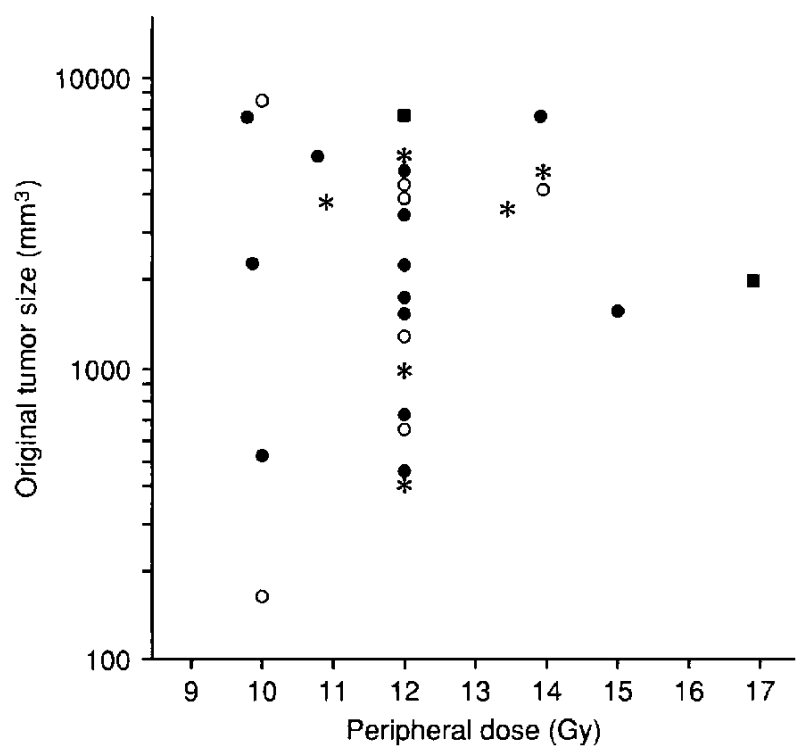

Fig. 2 Change of MR signal intensity, tumor shrinkage, original tumor size, and dose delivered to the tumor periphery. Closed circles show patients with MR signal intensity change in the central area of the tumor without tumor shrinkage. Asterisks show patients with tumor shrinkage with and without MR signal intensity change. Open circles show patients without tumor shrinkage or MR signal intensity change. Closed squares show patients with enlarging cyst in the tumor after treatment.

the signal change in the tumor and the dose delivered to the periphery or center. Even $10 \mathrm{~Gy}$ at the periphery induced a lowering of the MR signal intensity in the central area of the tumor (Fig. 2).

\section{Clinical study}

Follow-up examination found trigeminal neuropathy and facial weakness in only one patient and this was temporary. No patient developed lower cranial nerve paresis. Two patients had transient vertigo just after the treatment. Another two patients required implantation of a shunt because of developing hydrocephalus.

Hearing acuity was studied in 20 patients after radiosurgery. Eight patients were excluded, six were already deaf at treatment and two underwent subsequent surgical removal of the tumor. Hearing acuity was maintained at the pretreatment levels in $85 \%$ $(17 / 20)$ at 3 months, $80 \%(16 / 20)$ at 6 months, $72 \%$ $(13 / 18)$ at 9 months, $75 \%(12 / 16)$ at 12 months, $67 \%$ $(8 / 12)$ at 15 months, $60 \%(6 / 10)$ at 18 months, and $50 \%(2 / 4)$ at 24 months. Patient T.Y. had a pure 


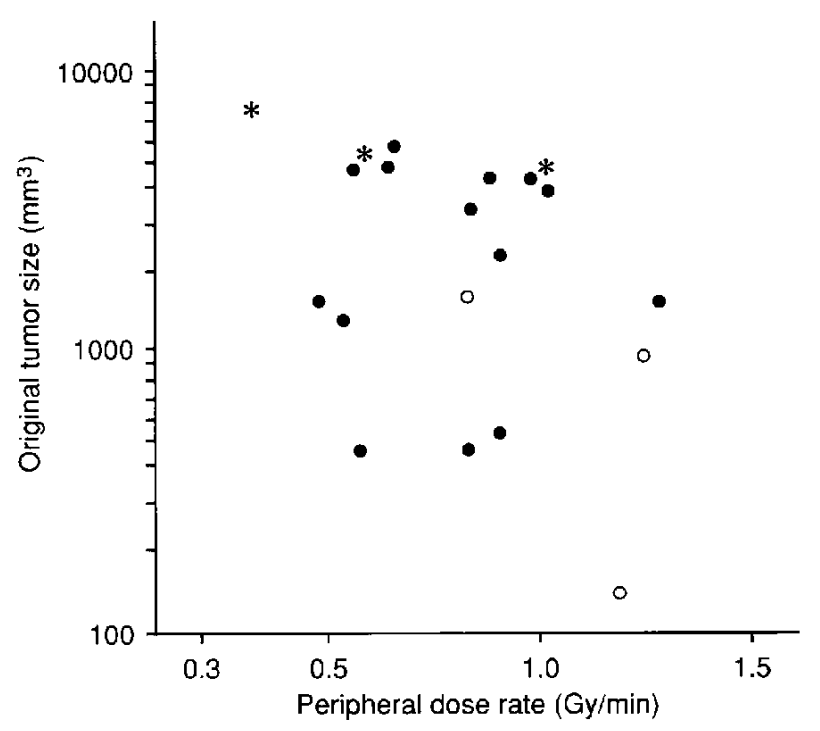

Fig. 3 Change of auditory acuity, original tumor size, and dose rate delivered to the tumor periphery. Open circles show patients with early decrease of hearing acuity after radiosurgery. Asterisks show those patients with late decrease. Closed circles show patients preserving hearing acuity.

tone average of $30 \mathrm{~dB}$ and speech discrimination score of $90 \%$ before treatment, and $28.8 \mathrm{~dB}$ and $85 \% 2$ years after treatment. Patient S.A. had $35 \mathrm{~dB}$ and $100 \%$ before treatment, and $43.8 \mathrm{~dB}$ and $60 \%$ after 15 months. Speech tone audiometry detected subtle changes of hearing.

The irradiation dose rate in the periphery was higher in patients with early hearing changes and the tumor was larger in patients with late changes (Fig. 3). A patient with neurofibromatosis type 2 showed late hearing change, although the dose rate was low. The hearing acuity was, however, already below the serviceable level before treatment. There was no definite correlation between the risk of hearing disturbance and the dose delivered to the periphery or center of the tumor. Even $10 \mathrm{~Gy}$ at the periphery induced hearing change. There was also no definite correlation with the length of the acoustic nerve irradiated.

Auditory function levels before treatment and during follow-up varied in the six patients with hearing disturbance after treatment (Fig. 4). Two of the six showed rapid decrease of hearing acuity within 3 months, and three showed a gradual decrease after 3 months. Interestingly, patient M.K. achieved recovery of hearing acuity after several months, from $16.3 \mathrm{~dB}$ and $100 \%$ before treatment, to $60 \mathrm{~dB}$

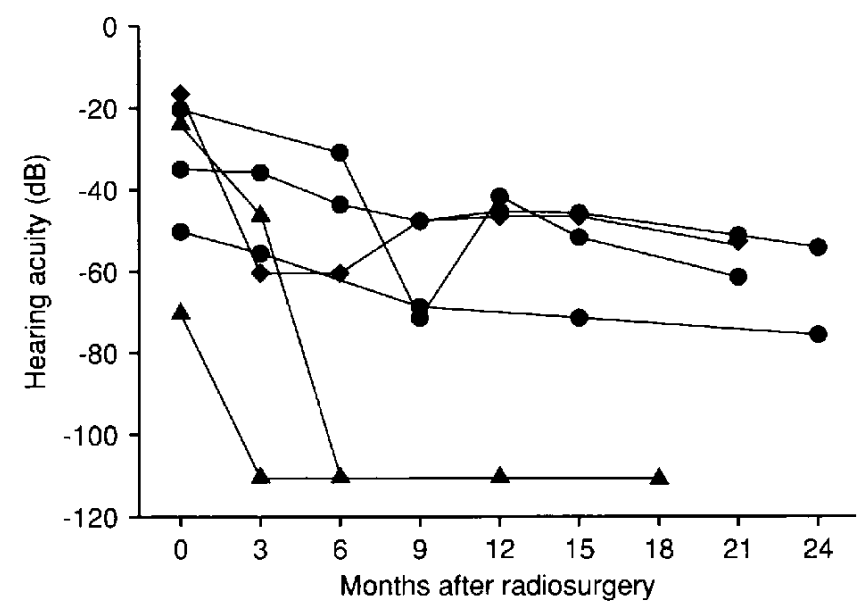

Fig. 4 Decrease of auditory acuity during followup. Triangles show patients with early decrease of auditory acuity. Circles show patients with late decrease. Diamonds show a patient with recovery of auditory acuity after initial decrease.

and $55 \%$ after 3 and 6 months, $46.3 \mathrm{~dB}$ and $100 \%$ after 9 months, $45 \mathrm{~dB}$ and $95 \%$ after 12 months, and $51.3 \mathrm{~dB}$ and $85 \%$ after 21 months. Ten patients still suffered from tinnitus after radiosurgery, worsening in some.

\section{Histological study}

The most prominent features of the tumor specimens were various vascular changes. There were fibrin deposits in the vascular wall and infiltration of macrophages and small lymphoid cells around the

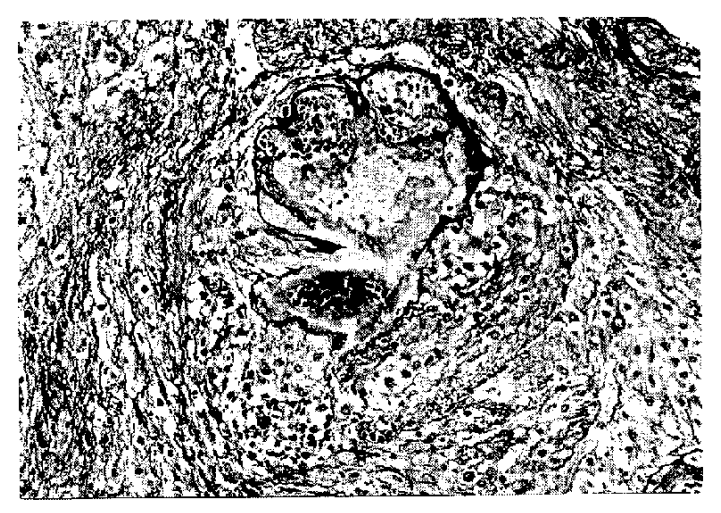

Fig. 5 Photomicrograph showing degradation of the basement membrane and thrombus formation. Periodic acid methenamine silver staining, specific for the basement membrane of the vascular wall, $\times 320$. 


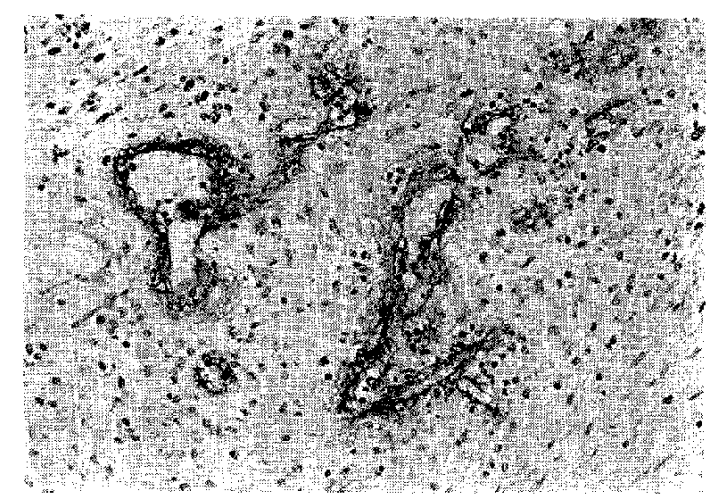

Fig. 6 Photomicrograph showing thickening of the vascular wall and endothelial proliferation. Factor VIII-related antigen staining, specific for the endothelium, $\times 320$.

vessels. Degradation of the basement membrane of the vascular wall and formation of thrombosis were present (Fig. 5). However, vascular proliferative changes were also seen as thickening of the vascular wall and endothelial proliferation (Fig. 6).

\section{Discussion}

Our study showed that gamma knife radiosurgery is effective for suppressing the growth of acoustic schwannoma with relatively low radiation doses, though the follow-up time was short. Previous reports found that high radiation doses ${ }^{6,7,9,10)}$ controlled tumor growth in $90 \%$ of cases. Low radiation doses $^{11,15)}$ ranging from 10 to $15 \mathrm{~Gy}$ achieved arrest of tumor growth in $81 \%$ of cases. Previous studies also found the MR signal intensity lowered in $70 \%$ of cases, and signs of shrinkage appeared in $30 \%$ of cases during the first 24 months after treatment. The MR imaging change rates in our study were slightly lower, but our patients were only followed up for a short time. As the beginning of signal change was within a period equivalent to that of other studies, the rate of change can be expected to increase later. Interestingly, doses ranging from 10 to $17 \mathrm{~Gy}$ at the periphery and from 17 to $34 \mathrm{~Gy}$ at the center all achieved lowering of the MR signal intensity. Larger tumors showed easier shrinkage and more rapid start of MR signal intensity changes. Our patients were treated with almost the same marginal dose, so these results suggest that the changes in the tumors occurred when the dose exceeded a threshold level rather lower than that previously considered. The histological study of surgical specimens 4 months after radiosurgery suggested that radiosurgery causes various changes in the vascular wall of the tumor, both destructive and proliferative. Acoustic schwannoma demonstrates various changes in vessels, such as ongoing thrombosis, hemorrhage, and angiogenesis, ${ }^{14)}$ so it is not clear which changes occurred as a result of radiosurgery. Nevertheless, the changes of the vascular wall in the tumor may be related to the MR signal intensity change or shrinkage of the tumor after radiosurgery. The radiobiological behavior of acoustic schwannoma in vivo is not well known, ${ }^{8)}$ so further study is needed to clarify the mechanism and the minimum dose for suppressing tumor growth."

Hearing preservation ${ }^{2)}$ has become the major target of treatment of acoustic schwannoma by both gamma knife radiosurgery and microsurgery. ${ }^{3,4)}$ In our study, $70-85 \%$ of patients maintained hearing at the pretreatment levels for 1 year, and about $50-60 \%$ beyond 1 year. Loss of useful hearing acuity began after 1 week to 12 months. ${ }^{7)}$ Comparison of hearing acuity at about 1 and 5 years showed the percentage of patients with unchanged hearing was about the same. ${ }^{11)}$ Therefore, our series shows that hearing acuity can be maintained for more than 2 years after treatment. Hearing disturbance was not significantly correlated with the marginal dose, central dose, or the length of acoustic nerve irradiated. The minimum dose delivered to the periphery, $10 \mathrm{~Gy}$, caused hearing disturbance. Therefore, the dose ${ }^{16)}$ or the length of cranial nerves irradiated, ${ }^{5)}$ suggested risk factors for peripheral nerve injury, were not significant predictors of cochlear nerve injury in this study. Tumor size and especially marginal dose rate might be correlated with hearing disturbance. The latter was higher in patients with hearing disturbance after treatment. This is also observed in patients with trigeminal neuropathy. As our patients were treated within the last 2 years and received almost the same marginal dose, a combination of collimators might be a primary factor. A combination of larger collimators used to plan the dosimetry results in a higher marginal dose rate. Therefore, our study reemphasizes the importance of precise dose planning to preserve hearing. For small schwannomas, especially those located in the internal auditory meatus, the collimator selection for the dose planning is very limited. More attention should be given to dose planning when treating very small schwannomas with the gamma knife. Classification of the auditory function before treatment and how to define serviceable hearing are also significant problems. ${ }^{2,4,12)}$

Cranial nerve injury, except for the cochlear nerve, was infrequent in our series. There was only one case each of trigeminal and facial neuropathy, 
and both were temporary. No patient showed lower cranial nerve paresis. This might be ascribed to the low marginal radiation dose or to precise dose planning. We always planned dosimetry on both MR images and CT scans three-dimensionally. The MR image provided a clear anatomical correlation between the tumor and critical structures such as the brainstem or cranial nerves, but distortion of the images was unavoidable. Comparing the dosimetry on both MR images and $\mathrm{CT}$ scans, especially at the porus of the internal auditory meatus, allowed correction of the distortion of MR images.

Hydrocephalus developed in two patients. The intratumoral cyst enlarged after treatment in another two patients. The mechanisms of hydrocephalus and cyst enlargement are unknown. Treatment of cyst enlargement remains difficult. We must beware of hydrocephalus and enlargement of intratumoral cyst after radiosurgery. ${ }^{7,11)}$

Relatively low dose gamma knife radiosurgery was effective in suppressing growth of acoustic schwannoma with preservation of hearing and low rates of complication. We can expect to obtain better treatment results with precise dose planning using $\mathrm{CT}$ and MR imaging, using an optimum dose and dose rate delivered to the periphery of the tumor.

\section{Acknowledgments}

The authors thank Mrs. M. Yokota, at Department of Pathology, Gunma University School of Medicine, for technical assistance of histochemical staining of surgical specimens of acoustic schwannoma.

\section{References}

1) Ganz JC, Mathisen JR, Thorsen F, Backlund E-O: Acoustic neurinoma: Early results related to radiobiological variables, in Lunsford LD (ed): Stereotactic Radiosurgery Update. New York, Elsevier, 1992, pp 359-363

2) Gardner G, Robertson JH: Hearing preservation in unilateral acoustic neuroma surgery. Ann Otol Rhinol Laryngol 97: 55-66, 1988

3) Glasscock ME III, Hays JW, Minor LB, Haynes DS, Carrasco VN: Preservation of hearing in surgery for acoustic neuromas. $J$ Neurosurg 78: 864-870, 1993

4) Kondziolka D, Lunsford LD: Preservation of hearing in acoustic neurinoma surgery. $J$ Neurosurg 78 : $154-156,1993$

5) Linskey ME, Flickinger JC, Lunsford LD: Cranial nerve length predicts the risk of delayed facial and trigeminal neuropathies after acoustic tumor stereotactic radiosurgery. Int $J$ Radiat Oncol Biol Phys 25: 227-233, 1993
6) Linskey ME, Lunsford LD, Flickinger JC: Radiosurgery for acoustic neurinomas: Early experience. Neurosurgery 26: 736-745, 1990

7) Linskey ME, Lunsford LD, Flickinger JC: Stereotactic radiosurgery for acoustic nerve sheath tumors, in Lunsford LD (ed): Stereotactic Radiosurgery Update. New York, Elsevier, 1992, pp 321-334

8) Linskey ME, Martinez AJ, Kondziolka D, Flickinger JC, Maitz AH, Whiteside T, Lunsford LD: The radiobiology of human acoustic schwannoma xenografts after stereotactic radiosurgery evaluated in the subrenal capsule of athymic mice. $J$ Neurosurg 78: $645-653,1993$

9) Noren G, Arndt J, Hindmarsh T: Stereotactic radiosurgery in cases of acoustic neurinoma: Further experiences. Neurosurgery 13: 12-22, 1983

10) Noren G, Arndt J, Hindmarsh $T$, Hirsch A: Stereotactic radiosurgical treatment of acoustic neurinomas, in Lunsford LD (ed): Modern Stereatactic Neurosurgery. Boston, Martinus Nijhoff, 1988, pp 481-489

11) Noren G, Greitz D, Hirsch A, Lax I: Gamma knife radiosurgery in acoustic neurinoma, in Steiner $\mathrm{L}$, Lindquist C, Forster D, Backlund E-O (eds): Radiosurgery: Baseline and Trends. New York, Raven Press, 1992, pp 141-148

12) Ogunrinde $\mathrm{OK}$, Lunsford LD, Flickinger JC, Kondziolka D: Stereotactic radiosurgery for acoustic nerve tumors in patients with useful preoperative hearing: Results at 2-year follow-up examination. $J$ Neurosurg 80: 1011-1017, 1994

13) Pollock BE, Lunsford LD, Kondziolka D, Flickinger JC, Bissonette DJ, Kelsey SF, Jannetta PJ: Outcome analysis of acoustic neuroma management: A comparison of microsurgery and stereotactic radiosurgery. Neurosurgery 36: 215-229, 1995

14) Sobel RA, Wang Y: Vestibular (acoustic) schwannomas: Histologic features in neurofibromatosis 2 and in unilateral cases. $J$ Neuropathol Exp Neurol 52: $106-113,1993$

15) Steiner L, Lindquist $\mathbf{C}$, Steiner $\mathrm{M}$ : Radiosurgery, in Symon L, Calliauw L, Cohadon F, Lobo Antunes J, Loew F, Nornes H, Pasztor E, Pickard JD, Strong AJ, Yasargil MG (eds): Advances and Technical Standards in Neurosurgery, vol 19. Wien, SpringerVerlag, 1992, pp 19-102

16) Urie MM, Fullerton $B$, Tatsuzaki $H$, Birnbaum $S$, Suit HD, Convery K, Skates S, Goitein M: A dose response analysis of injury to cranial nerves and/or nuclei following proton beam radiation therapy. Int $J$ Radiat Oncol Biol Phys 23: 27-39, 1992

Address reprint requests to: M. Hirato, M.D., Department of Neurosurgery, Gunma University School of Medicine, 3-39-15 Showa-machi, Maebashi 371, Japan. 